\title{
Bruce F. Pennington: Diagnosing Learning Disorders (Second Edition): A Neuropsychological Framework
}

\author{
Guilford Publications Inc., New York, NY, 355 pp, ISBN 978-1-59385-714-1, \$32.00 \\ (Hardcover)
}

\author{
Deborah K. Sokol
}

Published online: 1 September 2009

(C) The Author(s) 2009. This article is published with open access at Springerlink.com

This edition is a substantially revised version of the first edition by Dr. Bruce Pennington, drawing upon his thirty years of experience as a researcher, teacher, and practitioner in the field of developmental neuroscience. Indeed, this edition is compelling because of the author's major contributions and long active presence in the field. His experience uniquely enables him to integrate the past with present research findings and practice. Past achievements made within the field are interestingly chronicled and fully explained throughout the book to provide a foundation for the understanding of recent advancements. Findings from both clinical and basic science, particularly genetics, are synthesized to explain learning disorders including autism. Although the second edition largely features "evidence based" conceptualizations and applications, occasionally the author "goes out on a limb" by offering intellectually satisfying, empirically testable hypotheses to explain common problems. This can be done persuasively only by an experienced teacher who has a significant mastery of their field.

Diagnosing Learning Disorders-Second Edition can be utilized by practitioners (pediatricians, psychologists, therapists), researchers, and students to understand "the latest" in the burgeoning literature on learning disorders, including autism. The user can quickly look up a disorder, find the clinical description and comorbidities, learn about the candidate genes, and how the disorder has been characterized via neuroimaging and neuropsychological studies. Two neuropsychological profiles are presented and then prognosis and treatment are discussed. The book

D. K. Sokol $(\bowtie)$

Department of Neurology, Section of Pediatric Neurology, Indiana University School of Medicine, Indianapolis, USA

e-mail: dksokol@iupui.edu offers easy to use tables which concisely list the genetic studies and clinical summary data (symptoms, comorbidities, diagnosis, prognosis, and treatment) for each disorder: This information should be helpful to practitioners who must recognize developmental disorders, to psychologists when writing neuropsychological evaluations, and to already informed parents who wish to know more about their child's condition. The stated goals of this revision are to:

(1) Update the clinical description and research related to learning disabilities, emphasizing evidenced based interventions.

(2) Include additional disorders not featured in the original edition such as intellectual disability, mathematics disorder, and developmental coordination disorder

(3) Inform both practitioners and researchers including clinicians, professors, and students

(4) Extend the science of learning disorders into public health and educational policy

The author successfully achieves each of these goals via organizing the book into three sections: "Basic Concepts", "Reviews of Disorders" and "Implication for Practice and Policy". In "Basic Concepts" we are introduced to the "multiple deficit model" provided as an explanation for the frequently seen comorbid conditions such as reading disorders seen together with attention deficit-hyperactivity. These clinical observations are explained by this model via gene $x$ environment interactions or correlations that affect more than one neural system, producing "downstream effects" on the development of other neural systems. Affects may be deleterious or protective. For example, one observation repeated frequently in the book is the finding of poor short term verbal memory for children with 
language impairment and/or reading disorders that can also impair the learning of math facts. Another interesting observation, supported via cited research, is that deficits in processing speed occur for children with reading disorders and attention-deficit hyperactivity disorders, but not those with articulation (speech sound) disorders. Children with speech sound disorders, like those with reading disorders, have deficits in phonemic awareness. Therefore intact processing speed in children with speech sound disorders may in fact be protective against reading disorder for these children. This section includes a nice review of genetic and neuroanatomical and neuropsychological constructs. Further, there is an explanation of "less well validated" learning disorders commonly referred to by rehabilitation therapists such as central auditory processing disorder and sensory integration dysfunction. My only criticism in this section is that abbreviations for the disorders (RD, MD, SSD, LI, ID, DCD, CAPD, SMD) are often confusing.

A chapter is devoted to each of the following conditions in "Review of Disorders": dyslexia, speech and language disorders, autism spectrum disorder, attention-deficit/ hyperactivity disorder, intellectual disability, developmental coordination disorder, mathematics disorder and nonverbal learning disability. There is a robust description of each disorder which should engage a wide readership, along with detailed, updated description of genetic etiology, environmental influences, and brain mechanisms including recent neuroimaging research. Two case studies are then presented for each disorder utilizing (contemporary) neuropsychological test data for case conceptualization. Clinical and/or research summary tables are available at the end of each chapter.

The section on "Implications for Practice and Policy", at times seems overly expansive and impractical, e.g., suggesting the development of "an FDA-style national agency ...to set standards for the diagnosis, treatment, and education of children with learning disabilities"-p 262. However, this solution may now in fact fit the political climate! Further, the author bolsters his suggested broad policy change by compelling recent data about learning disorders' burden to society. After a description of evidence based interventions (with useful websites and resources in the appendix), there is a section on "controversial" therapies (e.g., auditory integration training, visual training) and how to recognize when they are harmful.

Compared to other books in this field, this book offers more evidence based interventions (when available) for the individual disorders. I recommend this for the intended audience (practitioners, researchers, and students), and also for already informed parents who wish to learn more about their child's condition.

Open Access This article is distributed under the terms of the Creative Commons Attribution Noncommercial License which permits any noncommercial use, distribution, and reproduction in any medium, provided the original author(s) and source are credited. 